\title{
Tratamento das hemorragias intracranianas espontâneas: o dilema continua
}

\section{Treatment of Spontaneous Intracranial Hemorrhages: the Dilemma Continues}

\author{
luri Santana Neville ${ }^{1}$ Djalma Felipe da Silva Menéndez ${ }^{1}$ Leonardo Moura Sousa Júnior ${ }^{1}$ \\ Eberval Gadelha Figueiredo ${ }^{2}$ Manoel Jacobsen Teixeira ${ }^{3}$
}
${ }^{1}$ Médico Neurocirurgião do Serviço de Neurocirurgia do Hospital das Clínicas da Faculdade de Medicina da Universidade de São Paulo (HCFMUSP), São Paulo, SP, Brasil
2 Médico Neurocirurgião Chefe do Grupo de Neurocirurgia vascular da Divisão de Clínica Neurocirúrgica; Supervisor da Divisão de Clínica Neurocirúrgica do HCFMUSP, São Paulo, SP, Brasil
3 Professor Titular e Chefe da Divisão de Clínica Neurocirúrgica do Departamento de Neurologia do HCFMUSP, São Paulo, SP, Brasil

\begin{abstract}
Address for correspondence luri Santana Neville, MD, Divisão de Clínica Neurocirúrgica do Departamento de Neurologia do HCFMUSP, rua Dr. Ovídio Pires de Campos, 171, ap. 618, Cerqueira César, São Paulo, SP, Brasil CEP 05403-010 (e-mail: iurineville@yahoo.com.br).
\end{abstract}

Arq Bras Neurocir 2015;34:291-294.

\section{Resumo}

\section{Palavras-Chave}

- hemorragia

- intracerebral

- intracraniana

- espontânea
Introdução As doenças cerebrovasculares (DCV) são a principal causa de morte no Brasil, sendo um grande problema de saúde pública em todo o mundo.

Métodos Revisão da literatura utilizando o banco de dados do MEDLINE. Buscados os termos "intracerebral" e "hemorrhage" presentes no título e no resumo publicados em qualquer data.

Resultados As hemorragias intracranianas (HIC) acometem, principalmente, os lobos cerebrais, gânglios da base, tálamo, tronco cerebral (predominantemente a ponte) e cerebelo como resultado da ruptura de vasos cerebrais afetados pelos efeitos degenerativos da hipertensão arterial sistêmica (HAS) ou da angiopatia amiloide. O diagnóstico pode ser feito através da tomografia computadorizada de crânio (TCC), sendo auxiliado pela ressonância nuclear magnética (RNM) do encéfalo e a angiografia dos quatro vasos cerebrais na investigação etiológica. Tratamento: dividido em clínico e cirúrgico. Atualmente, não há consenso sobre a seleção do tipo de tratamento para pacientes com HIC, e esta decisão deve levar em consideração idade do paciente, estado neurológico, tamanho e profundidade do hematoma, presença de hidrocefalia e de efeito compressivo. Os estudos atuais mostram uma tendência de superioridade dos procedimentos cirúrgicos minimamente invasivos, com menor risco de lesão encefálica secundária decorrente do acesso cirúrgico ao hematoma.

Conclusão A escolha da melhor estratégia para o tratamento das HIC permanece um desafio e ainda deve ser feita de forma individualizada. received

November 10, 2013

accepted

August 7, 2015

published online

October 19, 2015
DOI http://dx.doi.org/

10.1055/s-0035-1564888. ISSN 0103-5355.
Copyright $\odot 2015$ by Thieme Publicações License terms

Ltda, Rio de Janeiro, Brazil
(1)(1) $\Theta \circledast$ 


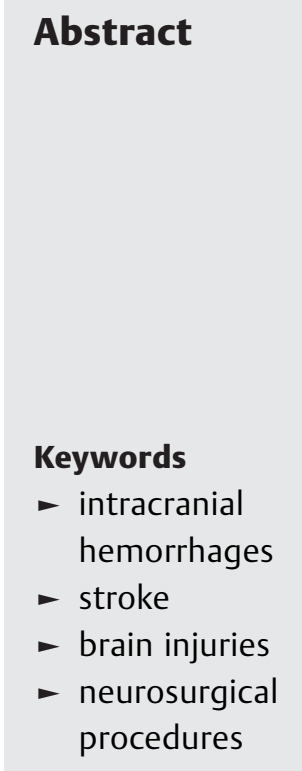

Introduction Cerebrovascular Diseases are the major cause of death in Brazil and a public health issue in the world.

Methods Review of the literature using the MEDLINE's data bank. We have searched the keywords "intracerebral" and "hemorrhages" in the title and abstract.

Results Intracranial hemorrhages (ICH) affect, mainly, the cerebral lobes, basal ganglia, thalamus, brain stem and the cerebellum as a result of the rupture of diseased cerebral vessels by the effects of hypertension or amyloid angiopathy. Diagnosis can be done with the use of a non-contrast computed tomography (CT), magnetic resonance imaging (MRI) and cerebral angiogram (useful in investigation of the etiology). Treatment can be divided in clinical and surgical. This decision still should be taken considering individual features, such as patient's age and neurological status, hematoma's size and deep, time between ictus and the procedure, presence of hydrocephalus and compressive effects. The studies has shown a preference for the minimally invasive procedures, since the secondary brain lesions caused by the surgery tend to be less.

Conclusion The choice of what would be the best strategy to treat the ICH is still a challenge and this decision should be taken individually.

\section{Introdução}

Os dados oficiais de mortalidade no Brasil revelam que a doença cerebrovascular (DCV) é a principal causa de morte, sendo responsável por mais óbitos do que a doença coronária nos últimos 40 anos, um fato que diferencia o nosso país dos demais no hemisfério ocidental (DATASUS). ${ }^{1}$

As hemorragias intracranianas (HIC) espontâneas, um dos subtipos de acidente vascular cerebral (AVC), são uma importante causa de morbidade e mortalidade no mundo, com taxas superiores às notadas nos outros subtipos de AVC.,3

Apesar da tendência declinante das taxas de mortalidade por doença cerebrovascular no país, a magnitude da doença é de grande importância, principalmente levando-se em consideração as outras consequências da doença cerebrovascular, como a invalidez, com alto custo social. Embora tenha ocorrido um aumento no número de internações de pacientes com HIC nos últimos 10 anos, a mortalidade permaneceu inalterada e ainda continua sendo uma condição catastrófica para os pacientes e seus familiares. ${ }^{1}$

\section{Métodos}

Realizada revisão da literatura utilizando o banco de dados do MEDLINE e a ferramenta de busca do PUBMED. Buscados os termos "intracerebral" e "hemorrhage" presentes no título e no resumo. Apenas os artigos escritos em inglês, realizados em humanos e publicados em qualquer data foram incluídos neste estudo.

\section{Resultados}

\section{Fisiopatologia}

As HIC podem ser classificadas em primárias ou secundárias (induzidas pelo uso de anticoagulantes), mas apresentam fisiopatologia semelhante. ${ }^{4}$ Acometem, principalmente, os lobos cerebrais, gânglios da base, tálamo, tronco cerebral (predominantemente a ponte) e cerebelo como resultado da ruptura de vasos cerebrais afetados pelos efeitos degenerativos da hipertensão arterial sistêmica (HAS) ou da angiopatia amiloide. Boa parte dos sangramentos ocorre próximo ou na bifurcação das artérias perfurantes que se originam dos grandes vasos intracranianos. ${ }^{3,5} 0$ sangramento que sucede à ruptura dos vasos cerebrais leva a uma lesão primária do tecido cerebral, decorrente da desconexão dos neurônios e das células da glia causada pelo efeito expansivo do hematoma, causando oligoemia, liberação de neurotransmissores (especialmente o glutamato), disfunção mitocondrial e despolarização da membrana neuronal. ${ }^{6-8}$ Uma cascata se sucede ao evento inicial, gerando uma série de lesões secundárias em decorrência do efeito tóxico dos produtos de degradação da coagulação e da hemoglobina, particularmente a trombina, que ativa a micróglia após 4 horas do íctus. ${ }^{9-12}$

\section{Diagnóstico}

Realizado através da tomografia computadorizada de crânio (TCC), que é capaz de mostrar a hemorragia como uma lesão hiperdensa logo após a sua ocorrência. ${ }^{13,14}$ A ressonância nuclear magnética (RNM) de encéfalo pode ser útil no sentido de identificar a etiologia da HIC. A arteriografia dos quatro vasos cerebrais deve ser realizada quando a suspeita de HIC secundária à ruptura de aneurisma cerebral ou de uma malformação arteriovenosa (MAV) é levantada. ${ }^{15} \mathrm{~A}$ recomendação é de que seja feita em todos os pacientes que não se enquadrem no perfil de HIC devido a HAS. Portanto, não está indicada aos pacientes com mais de 45 anos, previamente hipertensos e com HIC talâmica, putaminal ou em fossa posterior. Caso o resultado seja negativo para aneurisma cerebral ou MAV, o exame deve ser repetido após a absorção do hematoma, por volta de 2 a 3 meses. $^{2}$ 


\section{Tratamento}

O tratamento da HIC pode ser dividido em clínico e cirúrgico. O primeiro envolve medidas de suporte ventilatório, controle dos níveis pressóricos, da glicemia e de distúrbios metabólicos. O risco de deterioração neurológica e instabilidade cardiovascular é máximo nas primeiras 24 horas após o início dos sintomas, e portanto os pacientes devem ser mantidos em uma unidade de cuidados intensivos ou de tratamento de AVC. ${ }^{15}$ Diversos estudos já procuraram definir os níveis tensionais ideais para o tratamento destes pacientes na fase aguda, porque se por um lado os altos níveis tensionais podem aumentar o risco de expansão do hematoma, por outro, os baixos níveis pressóricos ocasionam a queda da pressão de perfusão cerebral, levando à isquemia secundária de regiões da chamada zona de penumbra. ${ }^{16-19}$

Já o tratamento cirúrgico consiste em: (1) monitoração da pressão intracraniana (PIC), (2) tratamento da hemorragia intraventricular (HIV), e (3) exérese do hematoma.

Atualmente, não há acordo sobre a seleção do tipo de tratamento para os pacientes com HIC, e a decisão sobre quando o tratamento cirúrgico seria o mais indicado permanece controversa. $^{20}$ Nota-se uma grande variabilidade nas taxas de cirurgias para o tratamento das $\mathrm{HIC}$, sendo realizadas raramente em países como a Holanda, porém alcançando até $50 \%$ dos pacientes em alguns centros na Alemanha e Japão. ${ }^{21}$ Na literatura, encontramos estudos randomizados (o primeiro foi publicado em 1961, na era pré-microscópio e pré-tomografia de crânio, e o mais recente, em 2006) comparando pacientes com HIC tratados de forma clínica ou conservadora. Os resultados são conflitantes, porém apresentam uma tendência a favorecer os pacientes tratados de forma cirúrgica na atualidade, com técnicas mais adequadas de neuroanestesia, bem como melhoria dos métodos diagnósticos e dos equipamentos utilizados na cirurgia.

\section{Tratamento Conservador}

Baseia-se nos achados de estudos prévios randomizados que não mostraram benefício no tratamento cirúrgico, possivelmente devido às lesões cerebrais adicionais advindas da manipulação de parênquima cerebral íntegro no acesso ao hematoma. ${ }^{22}$

\section{Tratamento Cirúrgico}

Aparentemente, os pacientes com hematomas supratentoriais de volume acima de $30 \mathrm{~mL}$ (porém menor do que $80 \mathrm{~mL}$ ), de localização lobar subcortical ( $<1 \mathrm{~cm}$ do córtex) ou putaminal, com compressão do tronco encefálico ou hidrocefalia obstrutiva, não comatosos ou em vigência de deterioração neurológica, e com delta tempo do íctus até a cirurgia inferior a 12 horas parecem beneficiar-se da cirurgia, já que os estudos sugerem que esta população apresenta resultados inferiores quando tratada clinicamente. $^{22-25}$ Já os pacientes com hematomas sem as características supracitadas apresentam melhores resultados quando tratados de forma conservadora.

\section{Monitoração da Pressão Intracraniana (PIC)}

Este recurso é útil em pacientes com o comprometimento do nível de consciência. A monitoração da PIC é capaz de identificar pacientes com risco de deterioração neurológica devido ao aumento excessivo da PIC. Representa um papel importante dentro das unidades de cuidados intensivos, orientando a terapêutica no sentido de manter uma pressão de perfusão cerebral entre 50 e $70 \mathrm{mmHg}^{26}$

\section{Técnicas de Drenagem do Hematoma}

A exérese do hematoma pode ser realizada através de três técnicas: (1) craniotomia clássica, (2) drenagem estereotática, e (3) drenagem através da neuroendoscopia.

Craniotomia: técnica clássica de exérese do hematoma intracerebral através de cirurgia aberta. Associada a maior risco de lesão encefálica secundária devido ao acesso cirúrgico ao hematoma. Atualmente existe uma tendência à utilização de craniotomias menores, minimizando o dano ao parênquima cerebral íntegro. ${ }^{21}$

Drenagem estereotática do hematoma: técnica minimamente invasiva, com grande utilidade para drenagem de hematomas profundos (ex: HIC putaminal). A sua eficácia foi comprovada em estudo randomizado. ${ }^{27}$ Pode ser realizada apenas com anestesia local, útil em pacientes com alto risco cirúrgico.

Tratamento endoscópico das HIC: por muito tempo considerada uma técnica investigacional, mas atualmente com eficácia comprovada em estudos randomizados, sendo o primeiro grande estudo publicado em $1989 .^{23}$ Considerada uma técnica minimamente invasiva, realizada através de pequena incisão na pele seguida de uma trepanação ou minicraniotomia. Permite a colocação de derivação ventricular externa (DVE) sob visualização direta nos casos em que os ventrículos são acessados durante a cirurgia para lavagem endoscópica e exérese do hemoventrículo. Na maioria dos casos, a exérese do hematoma é feita sem a identificação de um foco ativo de sangramento (cerca de $70 \%$ dos casos). ${ }^{24}$

\section{Discussão}

As DCV ocasionam um grande impacto na saúde pública em todo o mundo. Apesar do aumento do número de internações nos últimos anos, e dos grandes estudos acerca do assunto, a taxa de mortalidade ainda é muito alta, além do grande problema decorrente dos pacientes com sequelas neurológicas graves., ${ }^{1,21} \mathrm{O}$ tratamento permanence controverso, principalmente com relação à seleção dos pacientes que mais se beneficiariam do tratamento cirúrgico. Aparentemente, pacientes com as seguintes características teriam melhores resultados quando tratados cirúrgicamente, através da drenagem do hematoma:

1. delta T do ictus > cirurgia: até 12 horas;

2. status neurológico: não comatosos ou em plena deterioração neurológica;

3. localização: subcortical ( $<1 \mathrm{~cm}$ do córtex cerebral), lobar e putaminal;

4. volume: maior do que $30 \mathrm{~mL}$, porém menor do que $80 \mathrm{~mL}$;

5. hidrocefalia obstrutiva;

6. compressão do tronco cerebral.

As técnicas utilizadas para a drenagem do hematoma são basicamente três: (1) craniotomia, (2) drenagem 
estereotática, e (3) utilização da neuroendoscopia. Estudos randomizados já comprovaram a segurança e eficácia dos três métodos, e atualmente tem-se dado preferência para aqueles minimamente invasivos, com menor risco de lesão encefálica secundária. Os pacientes com piora no nível de consciência decorrente de hipertensão intracraniana podem beneficiar-se da monitoração da PIC.

\section{Conclusão}

As HIC ainda são catastróficas para os pacientes, seus familiares e para a saúde pública. Apesar dos avanços na medicina, a mortalidade permaneceu inalterada na última década. Vale ressaltar que a decisão sobre o melhor tratamento a ser adotado deve ser individualizada, já que os pacientes com HIC podem beneficiar-se do procedimento cirúrgico, desde que bem selecionados. Ainda, os estudos atuais mostram uma tendência da superioridade dos procedimentos minimamente invasivos, com menor risco de lesão encefálica decorrente do acesso cirúrgico ao hematoma.

\section{Referencias}

1 Lotufo PA. Mortalidade pela Doença cerebrovascular no Brasil. Rev Bras Hipertens 2000;4:387-391

2 Greenberg MS. Handbook of Neurosurgery, 6th edition. M-Login Brothers; 2005

3 Qureshi AI, Tuhrim S, Broderick JP, Batjer HH, Hondo H, Hanley DF. Spontaneous intracerebral hemorrhage. N Engl J Med 2001; 344(19):1450-1460

4 Steiner T, Rosand J, Diringer M. Intracerebral hemorrhage associated with oral anticoagulant therapy: current practices and unresolved questions. Stroke 2006;37(1):256-262

5 Takebayashi S, Kaneko M. Electron microscopic studies of ruptured arteries in hypertensive intracerebral hemorrhage. Stroke 1983;14(1):28-36

6 Graham DI, McIntosh TK, Maxwell WL, Nicoll JA. Recent advances in neurotrauma. J Neuropathol Exp Neurol 2000;59(8):641-651

7 Lusardi TA, Wolf JA, Putt ME, Smith DH, Meaney DF. Effect of acute calcium influx after mechanical stretch injury in vitro on the viability of hippocampal neurons. J Neurotrauma 2004;21(1):61-72

8 Qureshi AI, Ali Z, Suri MF, et al. Extracellular glutamate and other amino acids in experimental intracerebral hemorrhage: an in vivo microdialysis study. Crit Care Med 2003;31(5):1482-1489

9 Nakamura T, Xi G, Park JW, Hua Y, Hoff JT, Keep RF. Holo-transferrin and thrombin can interact to cause brain damage. Stroke 2005;36(2):348-352

10 Nakamura T, Keep RF, Hua Y, Nagao S, Hoff JT, Xi G. Iron-induced oxidative brain injury after experimental intracerebral hemorrhage. Acta Neurochir Suppl (Wien) 2006;96:194-198

11 Wagner KR, Packard BA, Hall CL, et al. Protein oxidation and heme oxygenase-1 induction in porcine white matter following intracerebral infusions of whole blood or plasma. Dev Neurosci 2002; 24(2-3):154-160

$12 \mathrm{Xi} \mathrm{G}$, Keep RF, Hoff JT. Mechanisms of brain injury after intracerebral haemorrhage. Lancet Neurol 2006;5(1):53-63
13 Fiebach JB, Schellinger PD, Gass A, et al; Kompetenznetzwerk Schlaganfall B5. Stroke magnetic resonance imaging is accurate in hyperacute intracerebral hemorrhage: a multicenter study on the validity of stroke imaging. Stroke 2004;35(2): 502-506

14 Kidwell CS, Chalela JA, Saver JL, et al. Comparison of MRI and CT for detection of acute intracerebral hemorrhage. JAMA 2004;292(15):1823-1830

15 Broderick JP, Adams HP Jr, Barsan W, et al. Guidelines for the management of spontaneous intracerebral hemorrhage: A statement for healthcare professionals from a special writing group of the Stroke Council, American Heart Association. Stroke 1999; 30(4):905-915

16 Anderson CS, Huang Y, Wang JG, et al; INTERACT Investigators. Intensive blood pressure reduction in acute cerebral haemorrhage trial (INTERACT): a randomised pilot trial. Lancet Neurol 2008;7(5):391-399

17 Oliveira-Filho J, Silva SC, Trabuco CC, Pedreira BB, Sousa EU, Bacellar A. Detrimental effect of blood pressure reduction in the first 24 hours of acute stroke onset. Neurology 2003;61(8): 1047-1051

18 Qureshi AI. Antihypertensive treatment of acute cerebral hemorrhage $($ ATACH): rationale and design. Neurocrit Care 2007;6(1): 56-66

19 Qureshi AI. Antihypertensive Treatment of Acute Cerebral Hemorrhage $(A T A C H)$ trial: International Stroke Conference; New Orleans, LA.2008; 20-22

20 Fernandes HM, Gregson B, Siddique S, Mendelow AD. Surgery in intracerebral hemorrhage. The uncertainty continues. Stroke 2000;31(10):2511-2516

21 Qureshi AI, Mendelow AD, Hanley DF. Intracerebral haemorrhage. Lancet 2009;373(9675):1632-1644

22 Mendelow AD, Gregson BA, Fernandes HM, et al; STICH investigators. Early surgery versus initial conservative treatment in patients with spontaneous supratentorial intracerebral haematomas in the International Surgical Trial in Intracerebral Haemorrhage (STICH): a randomised trial. Lancet 2005;365(9457): 387-397

23 Auer LM, Deinsberger W, Niederkorn K, et al. Endoscopic surgery versus medical treatment for spontaneous intracerebral hematoma: a randomized study. J Neurosurg 1989;70(4): 530-535

24 Kuo LT, Chen CM, Li CH, et al. Early endoscope-assisted hematoma evacuation in patients with supratentorial intracerebral hemorrhage: case selection, surgical technique, and long-term results. Neurosurg Focus 2011;30(4):E9

25 Pantazis G, Tsitsopoulos P, Mihas C, Katsiva V, Stavrianos V, Zymaris S. Early surgical treatment vs conservative management for spontaneous supratentorial intracerebral hematomas: A prospective randomized study. Surg Neurol 2006;66(5):492-501, discussion 501-502

26 Morgenstern LB, Hemphill JC III, Anderson C, et al; American Heart Association Stroke Council and Council on Cardiovascular Nursing. Guidelines for the management of spontaneous intracerebral hemorrhage: a guideline for healthcare professionals from the American Heart Association/American Stroke Association. Stroke 2010;41(9):2108-2129

27 Hattori N, Katayama Y, Maya Y, Gatherer A. Impact of stereotactic hematoma evacuation on activities of daily living during the chronic period following spontaneous putaminal hemorrhage: a randomized study. J Neurosurg 2004;101(3):417-420 